\title{
Influence of pressure-relief insoles developed for loaded gait (backpackers and obese people) on plantar pressure distribution and ground reaction forces
}

\author{
Marcelo Peduzzi de Castro ${ }^{\mathrm{a}, \mathrm{b}, \mathrm{c}, *}$, Sofia Abreu ${ }^{\mathrm{a}}$, Viviana Pinto ${ }^{\mathrm{d}}$, Rubim Santos ${ }^{\mathrm{b}}$, \\ Leandro Machado ${ }^{\mathrm{a}, \mathrm{c}}$, Mario Vaz ${ }^{\mathrm{c}, \mathrm{d}}$, João Paulo Vilas-Boas ${ }^{\mathrm{a}, \mathrm{c}}$ \\ a Center of Research, Education, Innovation and Intervention in Sport, School of Sport, University of Porto, Rua Dr. Plácido Costa, 91, \\ 4200-450 Porto, Portugal \\ ${ }^{\mathrm{b}}$ Activity and Human Movement Study Center/Department of Physiotherapy, School of Allied Health Science, Polytechnic Institute of Porto, Rua Valente \\ Perfeito, 22, 4400-330 Vila Nova de Gaia, Portugal \\ ${ }^{\mathrm{c}}$ Porto Biomechanics Laboratory, University of Porto, Rua Dr. Plácido Costa, 91, 4200-450 Porto, Portugal \\ ${ }^{\mathrm{d}}$ Institute of Mechanical Engineering and Industrial Management, Rua Dr. Roberto Frias, 400, 4200-465 Porto, Portugal
}

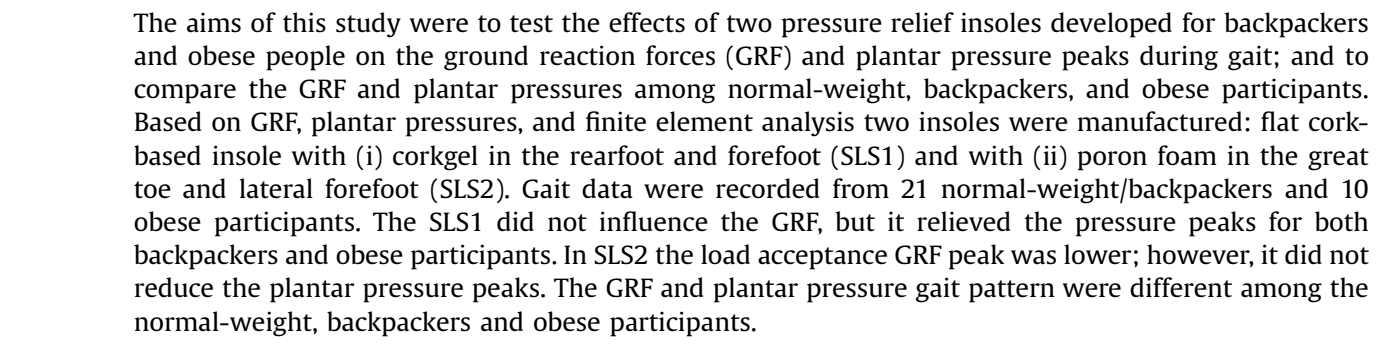

Keywords: Orthoses Obesity Walking

\section{Introduction}

The musculoskeletal system is often either permanently loaded, as in obese people, or occasionally loaded as in walkers that wear weighted backpacks (backpackers). In both cases (labeled as loaded populations), alterations in the biomechanical parameters of gait, such as in plantar pressure distribution (Castro et al., 2013; Castro et al., 2014; Hills et al., 2001) and ground reaction forces (GRF) (Birrell and Haslam, 2010; Birrell et al., 2007; Browning and Kram, 2007; Castro et al., 2013; Castro et al., 2014; Messier et al., 1996; Simpson et al., 2012) has been shown. Possibly, these biomechanical alterations may contribute to the higher incidence of low back pain (Grimmer and Williams, 2000; Skaggs et al., 2006), higher perceived exertion and shoulder discomfort (Simpson et al., 2011), second metatarsal stress fractures (Arndt et al., 2002), muscle strain
(Birrell and Haslam, 2009), joint problems (Birrell and Haslam, 2009), and foot blisters (Knapik et al., 1992) found in backpackers; and the loss of mobility (Messier et al., 1996), higher risk of hip and knee osteoarthritis (Felson, 1990; Hochberg et al., 1995; Ko et al., 2010), foot ulceration (Vela et al., 1998), and heel pain (Prichasuk and Subhadrabandhu, 1994) described in obese people.

Foot orthoses is a general term to describe a broad range of devices including heel lifts, lateral/medial wedges, or insoles (custom-made or prefabricated) (Chevalier and Chockalingam, 2012). These devices have been shown to be effective for managing many foot problems (Bonanno et al., 2011; Colagiuri et al., 1995; Cronkwright et al., 2011; Lynch et al., 1998; Sasaki and Yasuda, 1987). They can reduce and redistribute plantar foot pressure and subsequently avoid or decrease foot pain (Burns et al., 2007). However, the exact mechanisms by which foot orthoses work are yet to be fully understood (Chevalier and Chockalingam, 2012), and the biomechanical effect of these devices is far from the simplistic model often proposed in a clinical context (Nester et al., 2003); also, there is a need to establish the most suitable shoe/foot orthoses across clinical or high-risk populations (Rao et al., 2012). 
The comprehension of how the forces are distributed on the foot along the stance phase seems to be essential to detecting overloaded regions. The evaluation of the plantar pressures allows assessing the function of the ankle or foot while walking and other functional activities, as the foot and ankle are responsible for providing support and flexibility while weight transferring (Cavanagh and Ulbrecht, 1994). On the other hand, the plantar pressure systems do not provide any information regarding the shear forces. The analysis of the GRF provides global information about the vertical and shear stress forces during gait, whereas the plantar pressure analysis identifies the distribution of the vertical GRF over the plantar foot surface. The combination of both analyses offer more detailed information about specific features of forces acting on the foot during gait (Castro et al., 2013).

The knowledge of the different adaptations of the body when submitted to occasional or permanent load, and the development and testing of insoles developed specifically for these potential harmful situations may be helpful to further understand the mechanisms of foot orthoses to make physical exercise safe and to prevent injury. The primary aim of this study was to test the effects of two pressure relief insoles developed for loaded populations on GRF parameters and plantar pressure peaks during backpackers and obese' adults gait. The secondary aim was to compare the GRF and plantar pressures among normal-weight, backpackers and obese participants.

\section{Methods}

\subsection{Participants}

The participants were between 18 and 45 years old, had body mass index (BMI) either lower than 25 or higher than 30 and did not have any traumatic-orthopedic impairment or difficulty with independent gait. Twenty-one participants (10 men and 11 women; age $=25.81 \pm 2.47$ yrs; body mass $=63.62 \pm 6.96 \mathrm{~kg}$; height $=1.68 \pm 0.07 \mathrm{~m}$; and BMI $=21.56 \pm 3.65 \mathrm{~kg} / \mathrm{m}^{2}$ ) were selected as the normal-weight group. These participants wore loaded backpacks and they were also considered as backpackers group. Ten participants (five men and five women; ages $=35.60 \pm 4.90 \mathrm{yrs}$; body mass $=101.80 \pm 20.31 \mathrm{~kg}$; height $=1.66 \pm 0.10 \mathrm{~m}$; and $\mathrm{BMI}=36.50 \pm 4.51 \mathrm{~kg} / \mathrm{m}^{2}$ ) formed the obese group. This project was approved by the local ethical committee and all participants freely signed an informed consent term, based on Helsinki's declaration.

\subsection{Instruments and data acquisition}

A Bertec force plate, model 4060-15 (Bertec Corporation, Columbus, USA), operating at $1000 \mathrm{~Hz}$, and the Acknowledge software (BIOPAC System, California, USA) were used to capture GRF. The FScan in-shoe pressure system (TekScan, South Boston, USA) operating at $300 \mathrm{~Hz}$ with about 960 pressure cells (depending on the size of the shoe) with $0.18 \mathrm{~mm}$ thick insole sensor, and the F-Scan Research 6.33 software (TekScan, South Boston, USA) were used to capture plantar pressure data.

\subsection{Insoles}

We aimed to develop and test two models of insoles for an averaged "general" foot-not custom-made insoles-to be used indistinctly by people walking under loaded conditions (backpackers and obese subjects). Peak pressure relief was the rationale for the development of the insoles. Thus, based on the analysis of previous in-shoe plantar pressure data from backpackers (Castro et al., 2013) and obese subjects (Castro et al., 2014) during walking, as well as on simulations with a finite element model (FEM) which was adapted from a previous study (Pinto et al., 2011), the features of the insoles were selected for manufacturing and experimental testing.

\subsubsection{Finite element model (FEM) of the foot and insole}

From computed tomography medical images of one of the obese participants (male, with body mass of $121 \mathrm{~kg}$ ) was obtained a single file with the corresponding mesh clouds, using Mimics ${ }^{\circledR}$ v9.1 software (Materialise, Belgium). These mesh cloud were exported as STL files to Solidworks ${ }^{\circledR} 2009$ (Dassault Systèmes SolidWorks Corporation, Massachusetts, USA), where they were edited and improved to generate a solid part for each 3D object (further information about the FEM in Appendix 1).

For the insole construction model, a combined method of optical techniques (laser scanning method) and CAD model adjustments were used to obtain its geometry. Each obtained 3D insole model, with geometrical and material adjustments, was computationally tested at midstance gait cycle by a static simulation with the insole between the foot and the ground in the Ansys Workbench Platform ${ }^{\circledR}$ v.11 (Ansys, Pennsylvania, USA). The data were analyzed qualitatively (based on pressure distribution along the plantar surface) and quantitatively (based on values extracted from eight foot regions - Appendix 1).

After analyzing the FEM static simulation data, and the experimental gait data from backpackers (Castro et al., 2013) and obese subjects (Castro et al., 2014), two insoles were considered the most appropriate to reduce the pressure peaks. Thus, they were selected for manufacturing by a specialized company of shoe components: 3DCork Lda (Passos de Brandão, Portugal).

Both insoles were full-length, dual-density prefabricated (not customized), and had similar geometry (Fig. 1). They were labeled as stress-less shoe insole 1 (SLS1): flat insole made of cork (Young's Modulus $=1060 \mathrm{kPa}$ ) with corkgel A30 (Young's Modulus $=7.5 \mathrm{kPa}$ ) in the forefoot and rearfoot regions (Fig. 1); and as stress-less shoe insole 2 (SLS2): made of cork with poron foam (Young's Modulus $=63 \mathrm{kPa}$ ) in the great toe and lateral forefoot regions (Fig. 1). Unisex casual shoes with rubber sole (sneaker) with cutting and lining leather (Eject Shoes, Felgueiras, Portugal - Fig. 1), and its original insole (unisex flat insole made of polystyrene and leather lining) were used. The original insoles were removed from the shoes when testing the manufactured insoles.

\subsection{Tasks and procedures}

The participants' body mass and height were recorded. A cuff unit (VersaTek hub, F-Scan system) measuring $98 \times 64 \times 29 \mathrm{~mm}$ with Velcro strap up was attached on the lateral malleolus region of both legs of each participant. The participants were given a pair of thin socks and a sneaker with the sensor-pressure insole inside. This sneaker was selected due to its regular flat sole and a wide internal space. The participants familiarized with the setup by walking at a pace of 100 steps per minute, controlled by a metronome (Wittner Maelzel Metronome, Germany), over a 6 m walkway in which a force plate was embedded in the middle. For the testing, the obese participants performed three valid right foot trials in four conditions:

- Shoe-only condition (SHOE-ONLYCOND): wearing the sneakers without any insole;

- Original condition (ORIGINAL $\mathrm{O}_{\mathrm{COND}}$ ): wearing the sneaker with its original insole;

- SLS1: wearing the sneaker with the SLS1;

- SLS2: wearing the sneaker with the SLS2. 


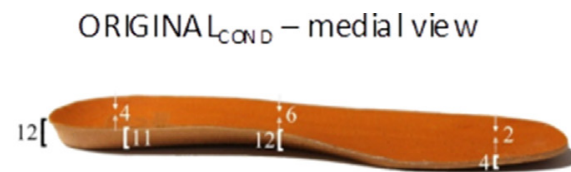

SLS1 - medial view

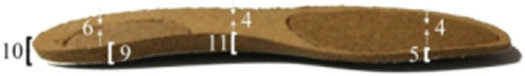

SLS2 - medial view

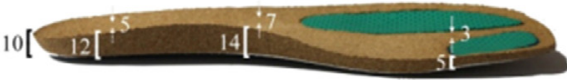

SLS1

superior view

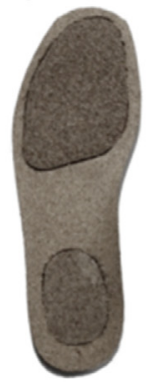

SLS1

FEM

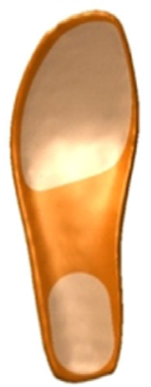

SLS2 superior view

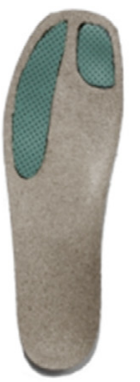

ORIGINAL

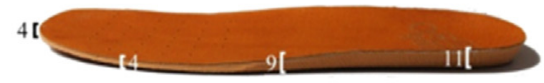

SLS1 - lateral view

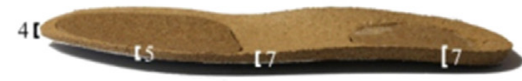

SLS2- medial view

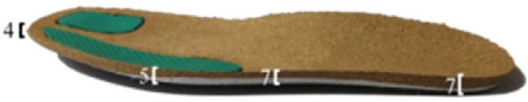

SLS2

CAD-FEM

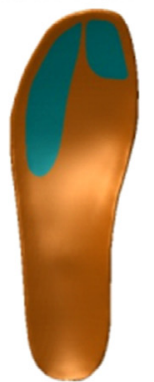

Shoes

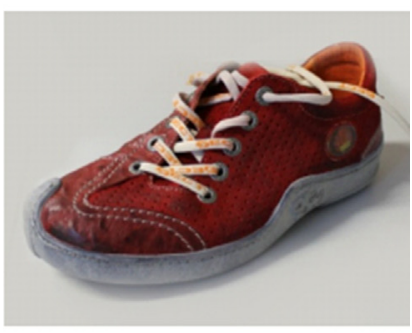

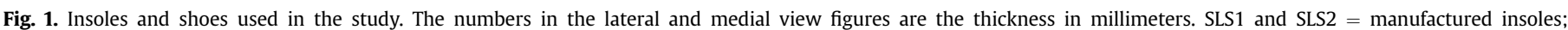
ORIGINAL

The normal-weight group performed three valid right foot trials wearing the sneakers without any insole, which was labeled as unloaded condition (UNLOADED ${ }_{\text {COND }}$ ). After that, the mass to raise their BMI to 30 was calculated, and a backpack was filled with sand and fixed at the central area of the participants' back (making them as backpackers group). The mass of the backpack ranged from 19.04 to $25.61 \mathrm{~kg}$ (mean load $22.26 \pm 1.44 \mathrm{~kg}$ ). As BMI = 30 is considered as a possible threshold for traumatic-orthopedic injuries (Class I obesity), we used it as load criterion to promote an occasional load (which is potentially harmful in the long-term) to assess load carriage during a standardized and substantially difficult (heavy) condition (Castro et al., 2013). Then they performed three right foot trial with the same four conditions as the obese participants. The order of the conditions was randomized.

\subsection{Data analysis}

The data from the force plate (three GRF components) and the in-shoe pressure system (values of each sensor in each frame) were exported to Matlab 7.0 software (MathWorks, Massachusetts, USA) and a program was developed for data processing and calculation of the variables.

Considering the absolute GRF data, the followed parameters were calculated:

- Duration of the stance phase;

- Fz1 (load acceptance peak): first peak from the vertical GRF;

- Fz2 (minimum between peaks): minimum value between the two peaks from vertical GRF.
- Fz3 (thrust peak): second peak from the vertical GRF;

- Fy1 (braking peak): first (negative) peak from the anteriorposterior GRF;

- Fy2 (propulsive peak): second peak (positive) of the anteriorposterior GRF;

- Fx (medial-lateral peak): peak from the medial-lateral GRF.

Regarding the pressure data, the program calculated the in-shoe pressure peak (sensor that showed the highest pressure value during the stance) for 10 foot regions: great toe, little toes, medial, central and lateral forefoot; medial and lateral midfoot; and medial, central and lateral rearfoot, as previously proposed (Castro et al., 2013).

\subsection{Statistical analysis}

Some variables were chosen arbitrarily to verify the intraindividual repeatability of the three trials. For this, the intra-class correlation coefficient (ICC) was calculated to the stance phase duration, Fz1, Fz3, and for the pressure peaks in the lateral forefoot and central rearfoot.

The mean of the three repetitions of each participant was computed and all the statistical procedures were performed with these mean values. To verify the influence of the insoles on the gait pattern, a repeated measures MANOVA was conducted with the groups (normal-weight/backpackers and obese participants) as between group factor, conditions (UNLOADED

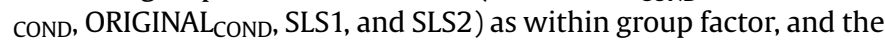
set of variables (GRF: duration of stance phase, Fz1, Fz2, Fz3, Fy1, 
Fy2, and Fx; or pressure peaks from the 10 regions) as dependent measures. The partial Eta square $\left(\eta_{\mathrm{p}}^{2}\right)$ was used to measure the effect sizes considering that an $\eta_{\mathrm{p}}^{2}$ of 0.01 was considered small, of 0.06 medium, and above 0.14 large (Stevens, 2002). The statistical analyses were performed using the Statistica ${ }^{\circledR}$ v.8 software (Statfoft ${ }^{\circledR}$, Tulsa, USA) with an $\alpha$ value set as 0.05 .

\section{Results}

Good to excellent data repeatability was found. All variables showed ICC higher than 0.89 .

\subsection{Differences in GRF among groups}

ANOVA interaction among conditions, groups and GRF variables $\left(F(24,696)=25.498 ; p<0.001 ; \eta_{\mathrm{p}}^{2}=0.468\right)$ with a large effect size was found. Similar stance phase durations $(p>0.05)$ among normal-weight $(0.74 \pm 0.03 \mathrm{~s})$, backpackers $(0.780 .03 \mathrm{~s})$, and obese participants $(0.77 \pm 0.05 \mathrm{~s})$ were found. The obese participants showed higher Fz1, Fz2 and Fz3 compared to backpackers and normal-weight participants, and higher Fy1 and Fy2 than normalweight participants. The backpackers showed higher Fz1, Fz2, Fz3, Fy1 and Fy2 than normal-weight subjects. Similar Fx were found among the groups (Fig. 2).

\subsection{Influence of insoles on the GRF}

Differences among conditions were found in GRF parameters for both backpackers and obese participants. Obese individuals presented lower Fz1 and Fz2 in SLS2 than in the other conditions. Backpackers also showed lower Fz1 in SLS2 than in ORIGINAL

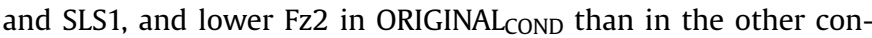
ditions. Similar values were found for Fz3, Fy1, Fy2 and Fx among conditions during obese and backpackers' gait (Table 1).

\subsection{Differences in plantar pressure peaks among groups}

ANOVA interaction among conditions, groups and pressure peaks $\left(F(36,1044)=2.138 ; p<0.001 ; \eta_{\mathrm{p}}^{2}=0.068\right)$ with a medium effect size was found. The obese participants showed higher pressure peaks in the central and lateral forefoot compared to normal- weight (UNLOADED COND) $_{\text {) }}$ and backpackers; and higher values in the medial forefoot, lateral midfoot and lateral rearfoot than normal-weight participants. The backpackers showed higher pressure peaks in the great toe, little toes, medial and central forefoot, and medial and central rearfoot than walking without a backpack (Fig. 3).

\subsection{Influence of insoles on plantar pressure peaks}

Significant differences among conditions were found in backpackers' gait. In the great toe and medial forefoot regions the SHOE-

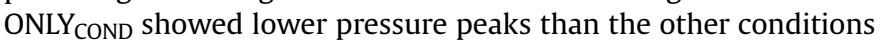
(ORIGINAL COND, SLS1 and SLS2). The SLS1 showed lower values in the little toes and medial midfoot regions and a higher value in the

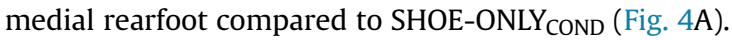

Differences among conditions in pressure peaks were also found for the obese participants. The SLS1 showed higher pressure peaks in the great toe, the SLS2 in the medial forefoot, and the SHOEONLY $_{\text {COND }}$ in the central forefoot compared to the other conditions. For the lateral forefoot and midfoot the pressure peaks in the SLS1

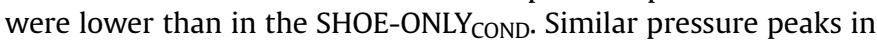
the rearfoot were found among conditions during obese' gait (Fig. 4B).

\section{Discussion}

The aims of this study were to compare the plantar pressure peaks and GRF among normal-weight, backpackers and obese participants, and to test the effects of two pressure relief insoles on these parameters during gait. The differences found among the groups (normal-weight, backpackers, and obese participants) and among conditions (SHOE-ONLY COND, ORIGINAL ${ }_{\text {COND, SLS1, and }}$ SLS2) for the backpackers and obese individuals were not only statistical significant, but also appear to have a practical relevance as medium to large effect sizes were found.

\subsection{Differences in GRF among groups}

All analyzed events of the vertical GRF (Fz1, Fz2, and Fz3) suggest a load-dependent behavior. As expected, the obese participants (mean weight: $102 \mathrm{~kg}$ ) showed higher values than the
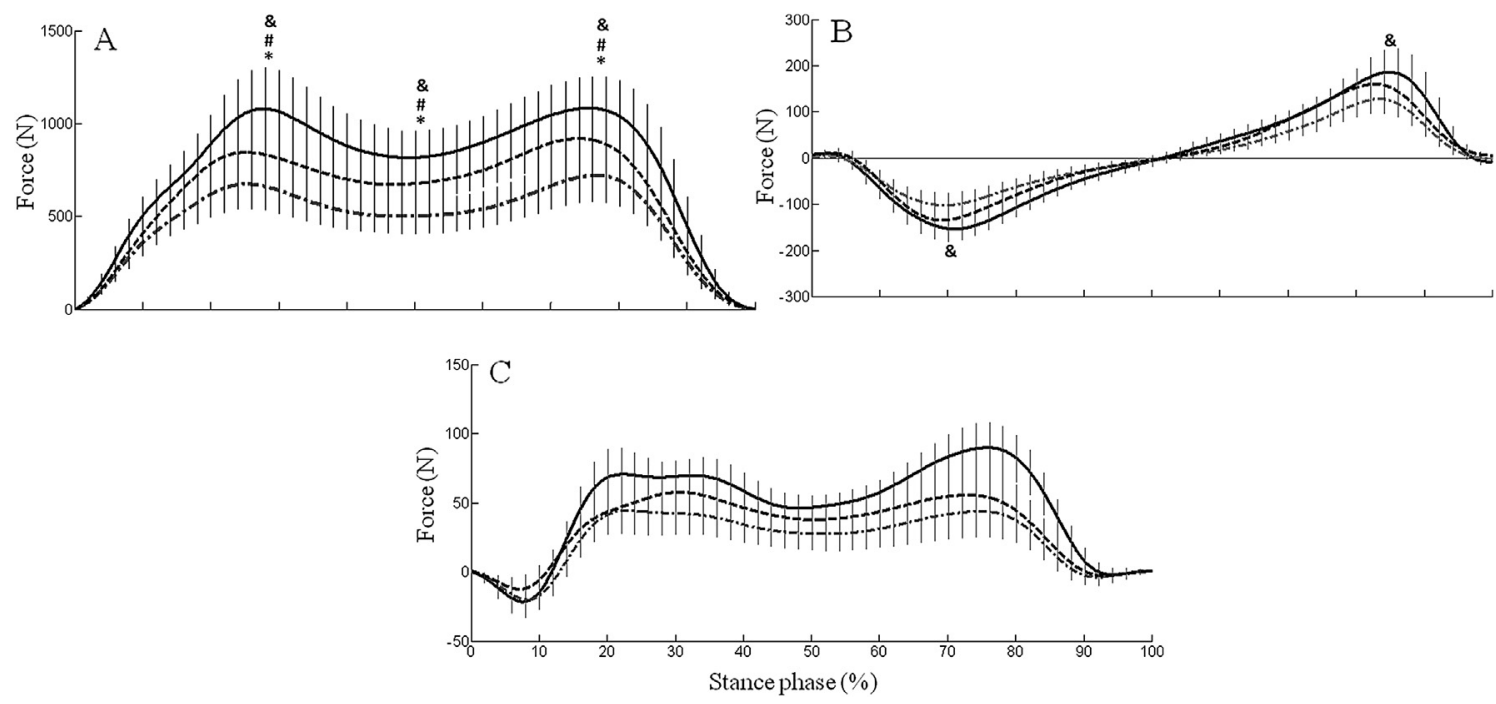

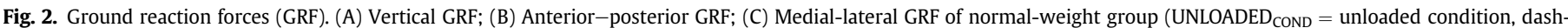

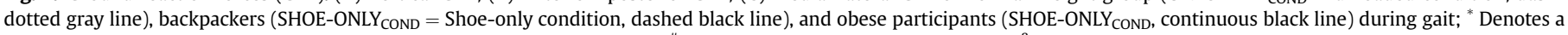
significant difference $(p<0.05)$ between obese and normal weight group; " between obese and backpackers; and ${ }^{\&}$ between backpackers and normal-weight group. 
Table 1

Ground Reaction Forces during backpackers and obese' gait in different conditions.

\begin{tabular}{|c|c|c|c|c|c|}
\hline & \multirow[t]{2}{*}{ Variables } & \multirow{2}{*}{$\frac{\text { SHOE-ONLY }_{\text {COND }}}{\text { Mean }(S D)}$} & \multirow{2}{*}{$\frac{\text { ORIGINAL }_{\text {COND }}}{\text { Mean }(S D)}$} & \multirow{2}{*}{$\frac{\text { SLS } 1}{\text { Mean (SD) }}$} & \multirow{2}{*}{$\frac{\text { SLS } 2}{\text { Mean (SD) }}$} \\
\hline & & & & & \\
\hline \multirow[t]{6}{*}{ Backpackers } & Fz1 $(\mathrm{N})$ & $860.0(118.6)$ & $868.0(113.7)^{*}$ & $863.3(118.5)^{\#}$ & $845.2(105.5)^{* \#}$ \\
\hline & $\mathrm{Fz} 2(\mathrm{~N})$ & $664.4(78.9)^{*}$ & $646.0(64.3)^{* \# \&}$ & $669.0(76.2)^{\#}$ & $665.5(78.1)^{\&}$ \\
\hline & Fz3 $(N)$ & $928.35(123.0)$ & $931.4(114.6)$ & $928.4(121.2)$ & 919.7 (103.3) \\
\hline & Fy1 (N) & $-141.5(11.9)$ & $-145.5(27.6)$ & $-140.7(23.9)$ & $-145.6(33.4)$ \\
\hline & Fy2 (N) & $157.8(29.6)$ & $159.0(26.4)$ & 153. 7 (30.3) & $151.1(24.1)$ \\
\hline & $\operatorname{Fx}(\mathrm{N})$ & $63.0(14.1)$ & $64.7(13.1)$ & $61.9(13.7)$ & $58.8(10.1)$ \\
\hline \multirow[t]{6}{*}{ Obese } & Fz1 $(\mathrm{N})$ & $1076.4(226.0)^{*}$ & $1074.8(233.8)^{\#}$ & $1078.4(227.3)^{8}$ & $1049.8(227.1)^{* \# \&}$ \\
\hline & Fz2 $(N)$ & $777.1(164.9)^{*}$ & $784.1(167.9)^{\#}$ & $777.8(171.8)^{\&}$ & $749.3(132.2)^{* \# \&}$ \\
\hline & $\mathrm{Fz} 3(\mathrm{~N})$ & $1052.2(197.0)$ & $1049.4(202.4)$ & $1060.0(195.4)$ & $1034.7(187.0)$ \\
\hline & Fy1 (N) & $-159.2(24.1)$ & $-166.8(25.5)$ & $-166.8(26.2)$ & $-165.0(25.7)$ \\
\hline & Fy2 (N) & $186.2(50.7)$ & $184.6(46.8)$ & $183.6(42.7)$ & $184.6(44.1)$ \\
\hline & $\mathrm{Fx}(\mathrm{N})$ & $91.5(18.3)$ & $94.9(17.8)$ & $95.2(19.3)$ & $94.0(20.1)$ \\
\hline
\end{tabular}

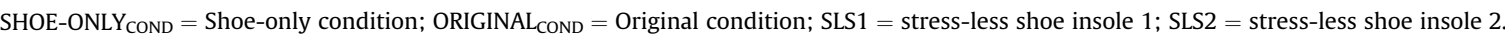

* \# \& Equal symbols between conditions denote significant difference with $p<0.05$.

backpackers (86 kg) and normal-weight (64 kg) group, and the backpackers showed higher values than the normal-weight group. Other studies comparing backpackers (Birrell and Haslam, 2010; Birrell et al., 2007; Castro et al., 2013; Simpson et al., 2012) and obese subjects (Browning and Kram, 2007; Messier et al., 1996) with normal-weight individuals corroborate with these findings. Considering the anterior-posterior GRF and being aware that higher magnitudes in this component have been related to blister development (Knapik et al., 1997). Our data suggest the obese participants may have developed gait pattern adaptations for preventing this kind of injury; while the backpackers seem to be more likely to develop blisters compared to normal-weight group, as evidenced by their higher anterior-posterior GRF peaks. These findings are in accordance with other studies (Birrell and Haslam, 2010; Castro et al., 2013; Simpson et al., 2012). In terms of medial-lateral GRF, no differences were found among the groups. As the medial-lateral GRF is related with gait stability (Birrell et al., 2007), these results were surprising because other studies assessing the GRF component in backpackers (Birrell et al., 2007; Castro et al., 2013; Simpson et al., 2012) or obese people (Browning and Kram, 2007), and kinematic studies (Lai et al., 2008; Qu, 2013) found greater instability in the loaded population' gait. We assessed the gait during overground level walking under a controlled pace. The aforementioned studies assessed the gait either with a self-selected speed (Birrell et al., 2007; Castro et al., 2013; Qu, 2013; Simpson et al., 2012), or with a controlled speed on a treadmill (Browning and Kram, 2007). These methodological criteria might cause these differences between the current study and others. Overall, our data support similar stability conditions among normal-weight group, backpackers and obese people walking over-ground at a controlled pace showed.

\subsection{Differences in plantar pressures among groups}

Only few studies have investigated the plantar pressure distribution during loaded gait (Birtane and Tuna, 2004; Castro et al., 2013; Hills et al., 2001). A previous study (Castro et al., 2013) found higher pressure peaks in nine out of ten foot regions in backpackers compared to a normal-weight condition (only the lateral midfoot showed similar values). In the present study higher values were observed in six out of ten regions. This differences may occurred because of the different shoes used in the studies: casual shoes with rubber sole (present study) versus ballet sneakers (Castro et al., 2013).

The obese participants presented an overall increased in the pressure peaks while walking compared to the normal-weight subjects. Two studies (Birtane and Tuna, 2004; Hills et al., 2001) assessed the plantar pressures in obese adults; in both of them, the rearfoot and midfoot were each considered as one region. Higher (Hills et al., 2001) and similar (Birtane and Tuna, 2004) values were found in the rearfoot, and the midfoot showed higher values (Birtane and Tuna, 2004; Hills et al., 2001) when compared to lean individuals. The present study partially agrees with them. We find higher values for the lateral region in both areas (rearfoot and

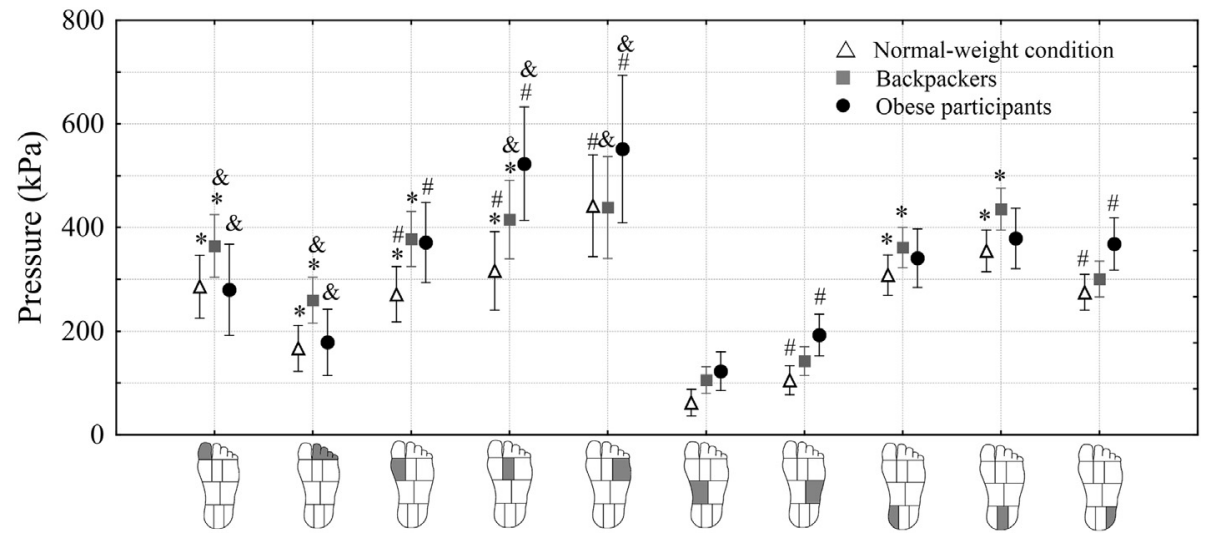

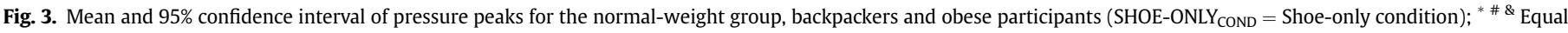
symbols denote significant differences $(p<0.05)$ between groups. 

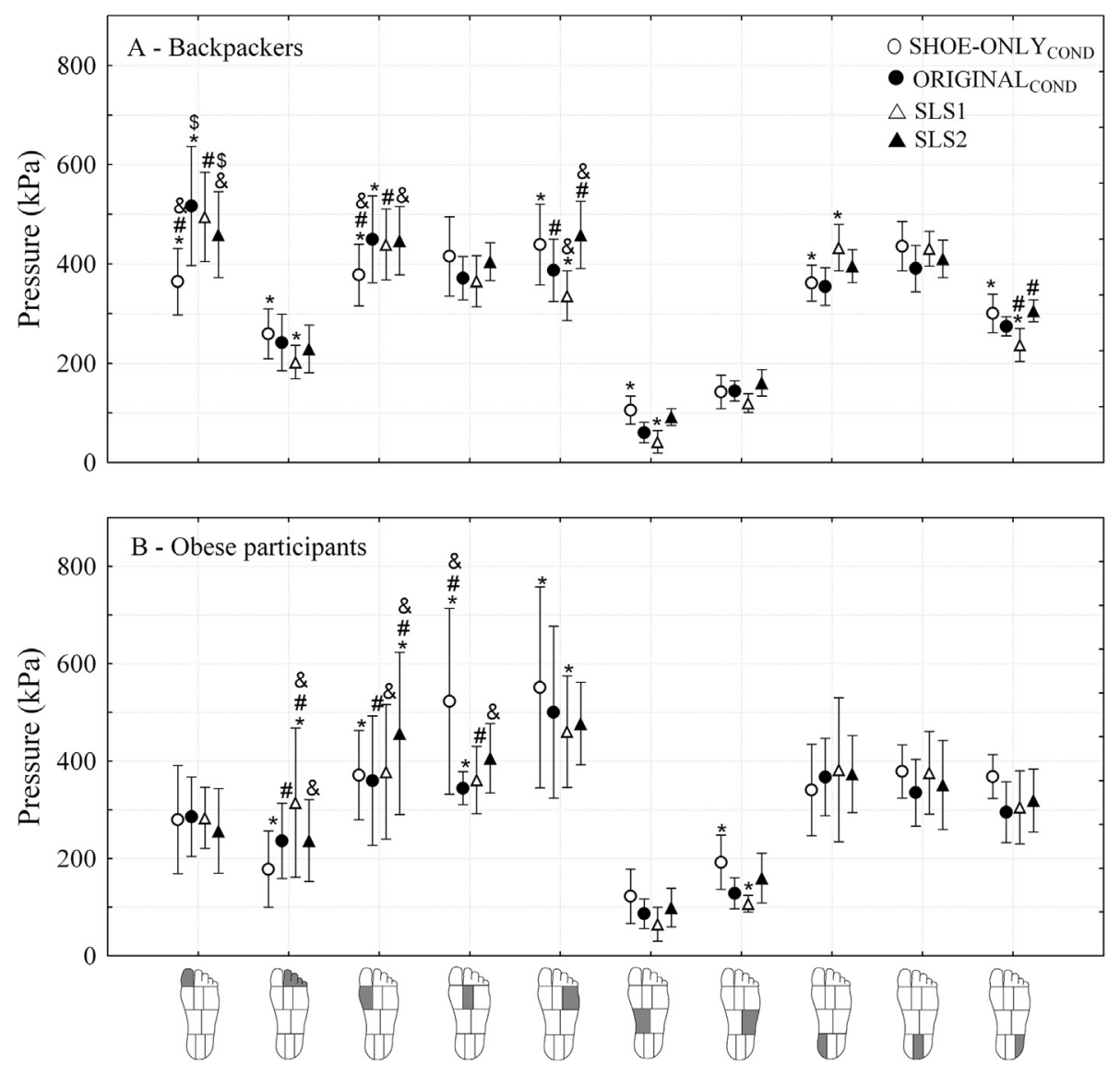

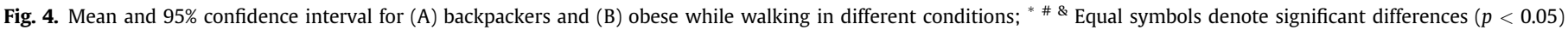
between groups.

midfoot), whereas similar values were found for the medial and central areas. The present study and a previous one (Hills et al., 2001) found increased pressure peaks in the three forefoot regions in obese participants compared to normal-weight peers. In contrary, another study (Birtane and Tuna, 2004) did not find any difference between these populations. The different levels of obesity may be the cause of these differences among the studies: $36.5 \mathrm{~kg} / \mathrm{m}^{2}$ (present study), $38.8 \mathrm{~kg} / \mathrm{m}^{2}$ (Hills et al., 2001), and $32.2 \mathrm{~kg} / \mathrm{m}^{2}$ (Birtane and Tuna, 2004).

When both loaded gait groups were compared (obese vs. backpackers), different pressure peaks were found. These findings suggest that the load distribution (obese: abdominal - men; gluteofemoral - women vs. backpackers: mid back) and the duration of loading (permanent vs. occasional) may play an important role on the plantar pressure distribution pattern.

\subsection{Influence of SLS1 on gait}

This insole did not influence the GRF parameters, but it did influence the pressure peaks. This condition caused different effects in the groups. In the backpackers: the rearfoot pressure in SLS1 shifted from the lateral to the medial region. This insole also showed an interesting effect in the lateral forefoot, in which it decreased the peaks compared to the other conditions. However, compared to the shoe-only condition, higher values were found in the great toe and medial forefoot. In the obese participants: it seems that the SLS1 is the most appropriated insole as it displayed the most consistent pressure-relieving in all forefoot (region in which the highest peaks were found) and lateral midfoot regions with this orthosis. Thus, a homogenous application of lowerdensity material (in the rearfoot and forefoot regions) seems to have a positive effect for relieving pressure peaks during loaded gait.

\subsection{Influence of SLS2 on gait}

The SLS2 promoted attenuation in Fz1compared to the other conditions for both backpackers and obese participants. Thus, this insole appears to play a relevant role in helping the heel in shock absorption. One possible explanation is the different material applied in the rearfoot region. In this case, the higher density of SLS2 may be more effective in load-acceptance attenuation when compared to the lower-density material of SLS1. However, a positive influence of this condition was not identified in the plantar pressure peaks for both groups: no alterations in the rearfoot, midfoot, and great toe regions were found; whereas, in the forefoot, the SLS2 increased the pressure peaks in the lateral region for the backpackers and in the medial region for the obese participants.

\subsection{Limitations of this study}

The findings of this study should be read considering several limitations. First, only the immediate biomechanical effects of the insoles were investigated; thus, the results may not reflect the long term changes such as the degradation of the insole material and the acclimatizing of the participants. Second, the participants wore standardized shoes which may not be representative of what they typically wear; however, as the shoes can influence the GRF and 
pressure parameters, this approach was adopted in order to decrease other sources of influence on the data. Third, the FEM is a static model, analyzing the midstance gait, and it was based on only one of the participant's morphology; nevertheless, we also analyzed experimental data of both populations (backpackers and obese) for establishing the insoles' features. Overall, we believe the mentioned limitations were either needed to make the methods consistent or they were minimized and did not have relevant influence in the results. The good to excellent observed data repeatability hints and supports the good internal consistency for the present methods.

In conclusion, we noted that insoles developed for the loaded population (backpackers and obese participants) showed positive effects for either the plantar pressure distribution or GRF parameters. The medium to large effect sizes observed suggest that these findings can be generalized for the loaded population. The SLS1 did not influence the GRF parameters but did influence positively the pressure peaks; it seemed to be the most appropriate insole for loaded gait population (mainly for the obese). The SLS2 helped the heel in shock absorption during the heel strike (lowered Fz1); however it did not show any positive influence on the plantar pressure distribution. Several differences in GRF and plantar pressure peaks were found among the normal-weight group, backpackers and obese participants.

\section{References}

Arndt, A., Ekenman, I., Westblad, P., Lundberg, A., 2002. Effects of fatigue and load variation on metatarsal deformation measured in vivo during barefoot walking. J. Biomech. 35, 621-628.

Birrell, S.A., Haslam, R.A., 2009. The effect of military load carriage on 3-D lower limb kinematics and spatiotemporal parameters. Ergonomics 52, 1298-1304.

Birrell, S.A., Haslam, R.A., 2010. The effect of load distribution within military load carriage systems on the kinetics of human gait. Appl. Ergon. 41, 585-590.

Birrell, S.A., Hooper, R.H., Haslam, R.A., 2007. The effect of military load carriage on ground reaction forces. Gait Posture 26, 611-614.

Birtane, M., Tuna, H., 2004. The evaluation of plantar pressure distribution in obese and non-obese adults. Clin. Biomech. 19, 1055-1059.

Bonanno, D.R., Landorf, K.B., Menz, H.B., 2011. Pressure-relieving properties of various shoe inserts in older people with plantar heel pain. Gait Posture 33, 385-389.

Browning, R.C., Kram, R., 2007. Effects of obesity on the biomechanics of walking at different speeds. Med. Sci. Sports Exerc. 39, 1632-1641.

Burns, J., Landorf, K.B., Ryan, M.M., Crosbie, J., Ouvrier, R.A., 2007. Interventions for the prevention and treatment of pes cavus. Cochrane Database Syst. Rev. 17.

Castro, M., Abreu, S., Sousa, H., Machado, L., Santos, R., Vilas-Boas, J.P., 2013. Ground reaction forces and plantar pressure distribution during occasional loaded gait. Appl. Ergon. 44, 503-509.

Castro, M.P., Abreu, S.A., Sousa, H., Machado, L., Santos, R., Vilas-Boas, J.P., 2014. Inshoe plantar pressures and ground reaction forces during overweight adults overground walking. Res. Q. Exerc. Sport, forthcoming.

Cavanagh, P.R., Ulbrecht, J.S., 1994. Clinical plantar pressure measurement in diabetes: rationale and methodology. Foot 4, 123-135.

Chevalier, T.L., Chockalingam, N., 2012. Effects of foot orthoses: how important is the practitioner? Gait Posture 35, 383-388.

Colagiuri, S., Marsden, L.L., Naidu, V., Taylor, L., 1995. The use of orthotic devices to correct plantar callus in people with diabetes. Diabetes Res. Clin. Pract. 28, $29-34$.

Cronkwright, D.G., Spink, M.J., Landorf, K.B., Menz, H.B., 2011. Evaluation of the pressure-redistributing properties of prefabricated foot orthoses in older people after at least 12 months of wear. Gait Posture 34, 553-557.

Felson, D.T., 1990. The epidemiology of knee osteoarthritis: results from the framingham osteoarthritis study. Semin. Arthritis Rheum. 20, 42-50.

Grimmer, K., Williams, M., 2000. Gender-age environmental associates of adoles cent low back pain. Appl. Ergon. 31, 343-360.

Hills, A.P., Hennig, E.M., McDonald, M., Bar-Or, O., 2001. Plantar pressure differences between obese and non-obese adults: a biomechanical analysis. Int. J. Obes. 25 , 1674-1679.

Hochberg M.C., Lethbridge-Cejku, M. Scott, WW. Reichle, R, Plato, C.C., Tobin, J.D., 1995. The association of body weight, body fatness and body fat distribution with osteoarthritis of the knee: data from the Baltimore longitudinal study of aging. J. Rheumatol. 22, 488-493.

Knapik, J.J., Ang, P., Meiselman, H., Johnson, W., Kirk, J., Bensel, C.W.H., 1997. Soldier performance and strenuous road marching: influence of load mass and load distribution. Mil. Med. 162, 62-67.

Knapik, J.J., Reynolds, K., Staab, J., Vogel, J., Jones, B., 1992. Injuries associated with strenuous road marching. Mil. Med. 157, 64-67.

Ko, S., Stenholm, S., Ferrucci, L., 2010. Characteristic gait patterns in older adults with obesity-Results from the Baltimore Longitudinal Study of Aging. J. Biomech. 43, 1104-1110.

Lai, P.P.K., Leung, A.K.L., Li, A.N.M., Zhang, M., 2008. Three-dimensional gait analysis of obese adults. Clin. Biomech. 23, S2-S6.

Lynch, D., Goforth, W., Martin, J., Odom, R., Preece, C., Kotter, M., 1998. Conservative treatment of plantar fasciitis. A prospective study. J. Am. Podiatr. Med. Assoc. 88, $375-380$.

Messier, S.P., Ettinger, W.H., Doyle, T.E., 1996. Obesity: effects on gait in an osteoarthritic population. J. Appl. Biomech. 12, 161-172.

Nester, C.J., van der Linden, M.L., Bowker, P., 2003. Effect of foot orthoses on the kinematics and kinetics of normal walking gait. Gait Posture 17, 180-187.

Pinto, V., Ramos, N., Vaz, M., Marques, M., 2011. 3D Modelling for FEM Simulation of an Obese Foot, Technology and Medical Sciences. CRC Press, pp. 19-22.

Prichasuk, S., Subhadrabandhu, T., 1994. The relationship of pes planus and calcaneal spur to plantar heel pain. Clin. Orthop. Relat. Res. 306, 192-196.

Qu, X., 2013. Effects of cognitive and physical loads on local dynamic stability during gait. Appl. Ergon. 44, 455-458.

Rao, S., Riskowski, J.L., Hannan, M.T., 2012. Musculoskeletal conditions of the foot and ankle: assessments and treatment options. Best Pract. Res. Clin. Rheumatol. 26, 345-368.

Sasaki, T., Yasuda, K., 1987. Clinical evaluation of the treatment of osteoarthritic knees using a newly designed wedged insole. Clin. Orthop. Relat. Res., 181-187.

Simpson, K.M., Munro, B.J., Steele, J.R., 2011. Effect of load mass on posture, heart rate and subjective responses of recreational female hikers to prolonged load carriage. Appl. Ergon. 42, 403-410.

Simpson, K.M., Munro, B.J., Steele, J.R., 2012. Effects of prolonged load carriage on ground reaction forces, lower limb kinematics and spatio-temporal parameters in female recreational hikers. Ergonomics 55, 316-326.

Skaggs, D., Early, S., D’Ambra, P., Tolo, V., Kay, R., 2006. Back pain and backpacks in School Children. J. Pediatr. Orthop. 26, 6.

Stevens, J., 2002. Applied Multivariate Statistics for the Social Sciences, fourth ed. Lawrence Erlbaum Associates, Mahwah NJ.

Vela, S.A., Lavery, L.A., Armstrong, D.G., Anaim, A.A., 1998. The effect of increased weight on peak pressures: implications for obesity and diabetic foot pathology. J. Foot Ankle Surg. 37, 416-420. 